\title{
Das Projekt „Kinder im Garten“- eine Modell-Bildungsstätte für Kinder im Alter von drei bis sechs Jahren
}

\author{
ULRIKE BRUNKEN \& CORNELIA KLAR
}

\begin{abstract}
Since february 2009, a novel educational program for children between three and six years of age is being established in the Palmengarten. The paper describes the facilities, concept and educational opportunities of this model project.

\section{Zusammenfassung}

Im Palmengarten Frankfurt gibt es seit Februar 2009 eine Modell-Bildungsstätte speziell für Kinder im Alter von drei bis sechs Jahren. Das Konzept, die Einrichtung der Räumlichkeiten sowie Inhalte und Abläufe der Bildungsangebote werden vorgestellt.
\end{abstract}

\section{Ideen und Start}

Im Februar 2009 begann im Palmengarten das Projekt „Kinder im Garten“, das sich mit seinem Programm-Angebot speziell an Kinder im Alter von drei bis sechs Jahren wendet. Ins Leben gerufen wurde dieses bundesweit einzigartige Projekt durch eine Kooperation zwischen den Kitas Frankfurt, Städtische Kinderzentren und dem Palmengarten Frankfurt am Main.

Basierend auf den Erfahrungen, die das Bildungsnetzwerk der Städtischen Kitas innerhalb seiner vielfältigen Kooperationen zu Museen, dem Palmengarten, dem Zoo, dem Stadtwaldhaus und anderen Bildungsinstitutionen gesammelt hatte, war der Wunsch nach einer dauerhaften Einrichtung innerhalb einer Bildungsinstitution entstanden. Sie sollte speziell für die Bedürfnisse von Kleinkindern konzipiert und eingerichtet werden und kontinuierlich von wöchentlich wechselnden Kita-Gruppen besucht werden können.

Als im Haus Leonhardsbrunn nach dem Auszug der Berufsschule Räume frei geworden waren, eröffnete sich hier eine neue NutzungsMöglichkeit. Der Ostflügel des unter Denkmalschutz stehenden Gebäudes konnte dem Projekt mit zwei großen Aktionsräumen und einer $\mathrm{Au}-$ ßenfläche von ca. $1000 \mathrm{~m}^{2}$ zur Verfügung gestellt werden. Allerdings mussten zusätzliche Gelder für das Modell-Projekt gewonnen werden. Erst als drei Stiftungen (Deutsche Bundesstiftung Umwelt, Stiftung Flughafen Frankfurt/Main für die Region, Stiftung Polytechnische Gesellschaft Frankfurt am Main) die Zusage gaben, das Projekt für eine Start-Phase von drei Jahren insge- samt zur Hälfte mitzufinanzieren, konnte es in die Tat umgesetzt werden.

\section{Konzept}

Das Modell-Projekt zeichnet sich durch mehrere Besonderheiten aus: Wesentlich ist das interdisziplinäre Team, das aus einer Erzieherin, einer Sozialpädagogin, einer Gärtnerin und einer Biologin zusammengesetzt ist. Die Themen der Bildungsangebote werden gemeinsam erarbeitet und durchgeführt, so dass die gegenseitige Ergänzung optimal ist. Eine weitere Besonderheit ist die Dauer der Bildungsangebote für diese $\mathrm{Al}$ tersstufe. Im Gegensatz zu den sonst üblichen ein- bis zweistündigen Führungen in Museen oder anderen Bildungseinrichtungen können uns die Kindergruppen zu einem Thema an drei aufeinanderfolgenden Tagen von 9 bis $16 \mathrm{Uhr}$ besuchen. So haben die Kinder Zeit, sich auf ein Thema einzulassen und Schritt für Schritt neue Informationen dazu zu sammeln. Im Gegensatz zu den begrenzten Möglichkeiten bei einer relativ kurzen Führung ist es unser Ziel, dass die Kinder dabei möglichst viel selbst und eigenbestimmt entdecken und erlernen können.

Da die Konzentrationsfähigkeit der Kinder bedingt durch ihr Alter, Sprachverständnis und/ oder ihren Entwicklungsstand unterschiedlich ist, wecken wir ihre Aufmerksamkeit und Interesse durch häufigen Methodenwechsel immer wieder neu: Zuhören, beobachten, erfühlen, eigenständiges und gemeinsames Entdecken sowie freies und gemeinsames Spielen wechseln sich ab orientiert an den Bedürfnissen und Fähigkeiten der Kinder. Damit die Kleinkinder sich in den 


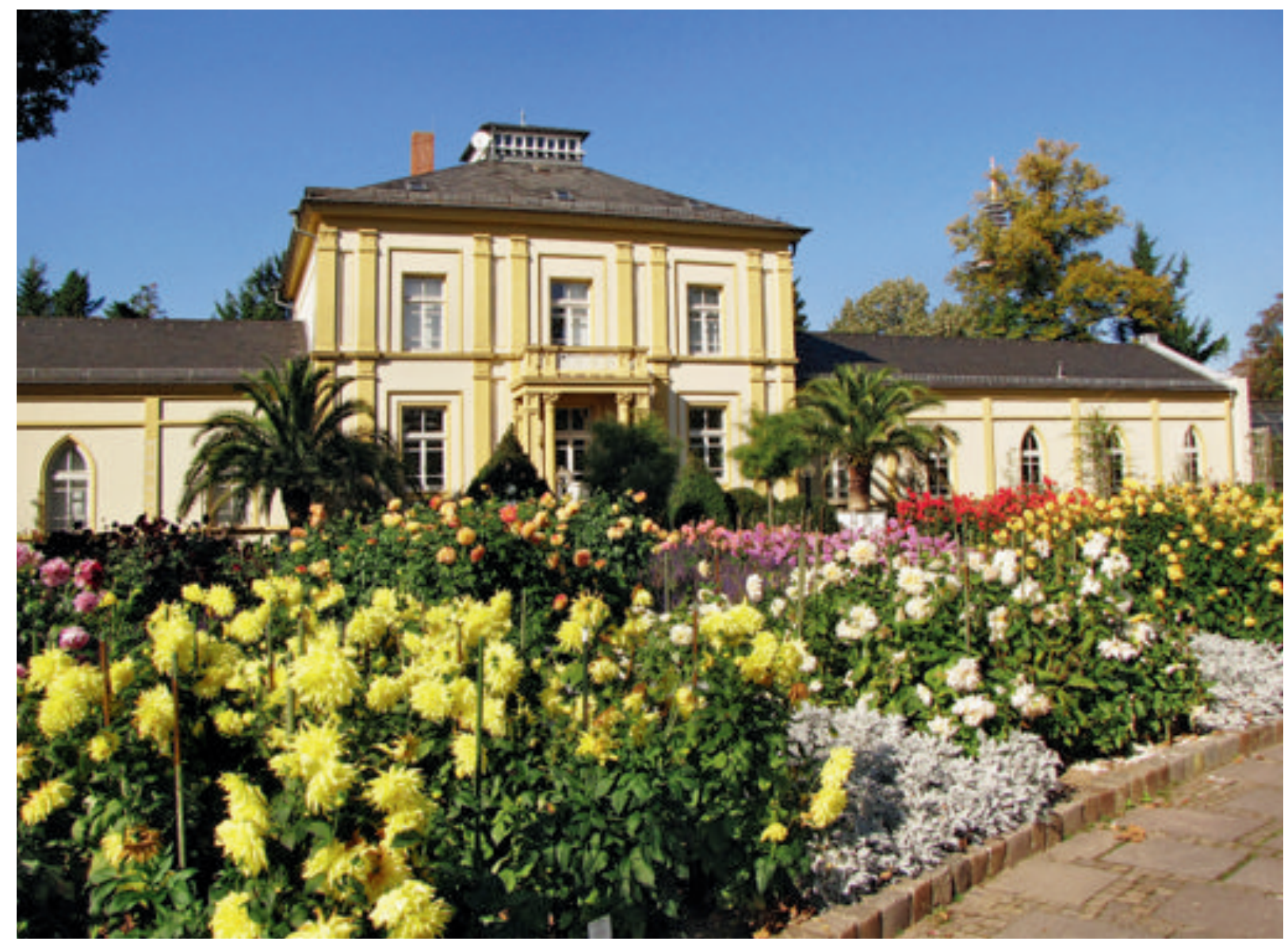

unbekannten Räumlichkeiten wohl fühlen, wurden bei der Einrichtung bekannte Einrichtungselemente aus dem Kita-Bereich (z. B. kindgerechtes Mobiliar und Sanitär-Einrichtung, Spielund Bauecke, Spielschränke mit bekanntem Spielmaterial) angeschafft und durch unbekannte Einrichtungselemente aus dem naturwissenschaftlichen Bereich erweitert. Hierdurch soll kindliche Neugier geweckt werden. Dazu gehört eine Forscher-Station mit unterschiedlichen Untersuchungsgeräten wie Lupen, Binokularen, Behältern, Trichtern, Pinzetten und Pipetten. Auch eine kleine Forschersammlung mit vielfältigen pflanzlichen und tierischen Materialien sowie kindgerechte Fachbücher aus dem Gebiet Biologie und Botanik stehen den Kindern zur Verfügung.

\section{Themen der Bildungsangebote}

Auch hinsichtlich der Themen versuchen wir neue Wege zu gehen. So ist es ein Ziel, im Sinne einer Bildung für nachhaltige Entwicklung ein Thema von seiner ökologischen, ökonomischen, sozialen und kulturellen Seite zu entdecken. Kernthema ist bei jedem Bildungsangebot eine bestimmte Pflanzengruppe. Wir erkunden mit den Kindern zuerst, wie diese Pflanzen aussehen und woran man sie erkennen kann. Als nächsten Schritt geben wir den Kindern einen Eindruck davon, wie und wo die Pflanzen am Naturstandort leben, wie sie wachsen und was sie zum Leben brauchen. Wir gehen auch darauf ein, welche Tiere von und mit den Pflanzen leben, so dass wir die Pflanze in ihren ökologischen Zusammenhängen darstellen. Als dritten Schritt besprechen wir mit den Kindern die Bedeutung der Pflanzen für uns Menschen, was aus ihnen hergestellt wird und vor allem auch auf welche Weise. Dabei kommen wir mit den Kleinkindern im Sinne der Bildung für nachhaltige Entwicklung auch auf verschiedene Probleme zu sprechen, so z. B. anhand der Rodung von Wäldern zur Gewinnung von Bauland oder Aufforstung

Abb. 1: Haus Leonhardsbrunn. In seinem rechten Flügel ist das Kinderprojekt untergebracht. 
von Plantagen, und diskutieren mit den Kindern auch darüber. So wird z. B. von STOLTENBerG (2008) gefordert: „Die Orientierung am Erhalt der Natur als Lebensgrundlage für Mensch und Kreaturen als ethisches Prinzip kann sich erweisen, indem man die Wahrnehmung und das Verständnis dafür entwickelt, dass wir in allen unseren täglichen Verrichtungen und in der Nutzung aller Produkte auf natürliche Lebensgrundlagen angewiesen sind und zurückgreifen. Sie aber wahrnehmen zu können, setzt Aufgeschlossenheit und auch Wissen über Wirkungszusammenhänge voraus."

Bislang werden regulär drei Themen angeboten: „Bambus" und „Palmen“ als Drei-TagesAngebote sowie „Wasserpflanzen“" als Ein-TagesAngebot. Ab 2011 bieten wir zudem als DreiTages-Angebot das Thema „Kletterpflanzen“ und als Ein-Tages-Angebot „Beerenobst“ an.

Wie nun die praktische Umsetzung dieser unterschiedlichen Aspekte und Anforderungen aussieht, wird nachfolgend am Beispiel des DreiTages-Angebotes „Bambus“ dargestellt.

\section{Drei-Tages-Angebot „Bambus“ im Projekt „Kinder im Garten“}

\subsection{Erster Tag}

Nachdem die Gruppe gegen 9 Uhr bei uns eingetroffen ist und dabei oft schon eine weite Strecke per Bus oder Bahn zurückgelegt hat, können die Kinder bei uns zuerst in Ruhe frühstücken. Danach treffen wir uns in der Gesprächs-Ecke, einer mit Teppich und Sitzkissen ausgestatteten Ecke. Dieser Platz wird in den nächsten drei Tagen immer Treffpunkt unserer gemeinsamen Gespräche sein. Dort stellen wir uns gegenseitig vor und besprechen mit den Kindern, wie die drei Tage ungefähr ablaufen werden. Anhand eines Tagesplans mit Bildsymbolen können die Kinder sehen, wie der Tag strukturiert ist. Dann gehen wir näher auf das Thema ein. Wie sieht Bambus aus? Woran kann man ihn

Abb. 2 (oben): Einstiegsrunde zum Thema Bambus. Die Kinder erforschen verschiedene Halme und Äste.

Abb. 3 (Mitte): Forscherreise zum Bambuswald im Palmengarten.

Abb. 4 (unten): Gemeinsames Kochen mit den Kindern.
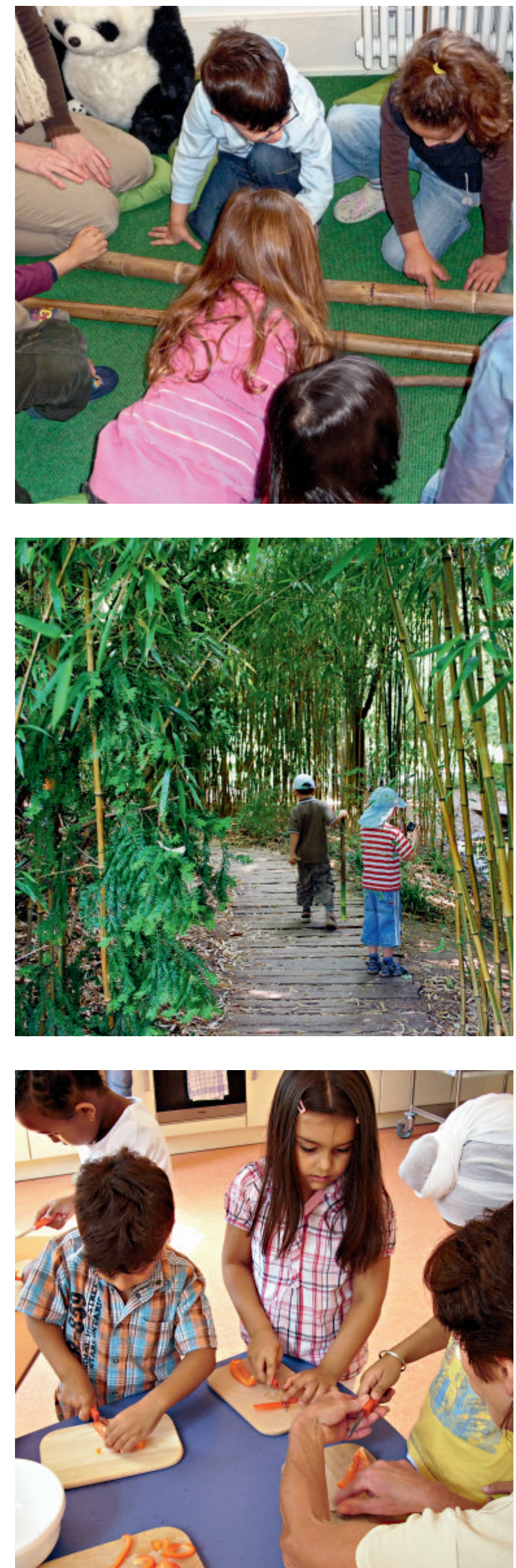

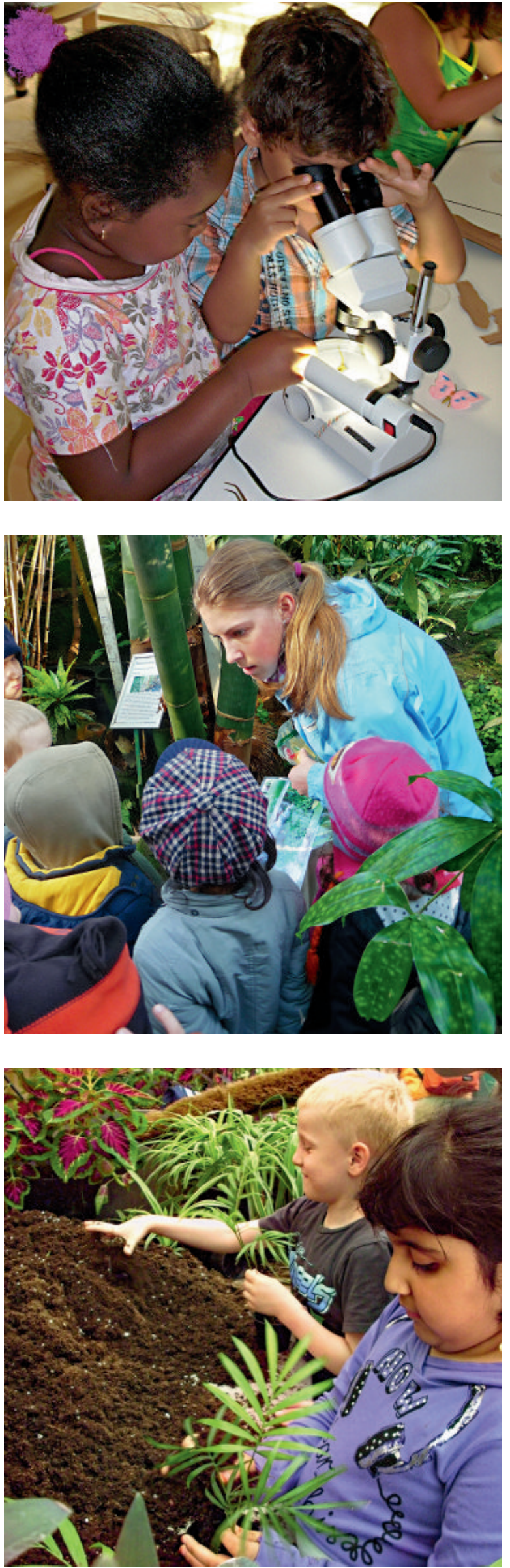

erkennen? Dies erforschen wir anhand dreier Stöcke - einem Bambushalm, einem Palmenstamm und dem Ast eines Baumes. Es ist faszinierend, wie interessiert und wissbegierig die Kinder schon hinsichtlich dieser drei Stöcke sind. Wir sehen, erfühlen und erfassen, ob die Stöcke rau oder glatt, leicht oder schwer, weich oder hart, hohl oder massiv sind, oder ob sie durch besondere Kennzeichen, wie ringartige Knoten, gegliedert sind. Erstaunt stoßen die Kinder mit ihren Fingern im hohlen Halm an eine Wand immer dort, wo man außen einen Knoten erkennen kann. Wir kommen zu dem Schluss, dass man Bambus an den durch ringartige Knoten gegliederten, glatten, innen zumeist hohlen Halmen erkennt, die hart wie Holz sind. Außerdem sehen wir uns die Blätter an, die lang, spitz und grün sind.

Anschließend gehen wir auf „Forscher-Reise“ in das Freigelände des Palmengartens. Die Kinder erhalten als ersten Forscher-Auftrag, einen Bambuswald zu suchen. Unterwegs schauen wir uns aber auch alles an, was am Wegesrand interessant ist - dies können Eichhörnchen, Vögel, Ameisen und Regenwürmer sein, die unseren Weg kreuzen, aber auch Gärtnerinnen und Gärtner bei unterschiedlichen Tätigkeiten, verschiedene Brunnen, Statuen, Bewässerungsanlagen und Kleinfahrzeuge. Gemeinsam beobachten wir und suchen nach Antworten auf entstehende Kinderfragen. Auf den Wegen Liegendes darf von den Kindern gesammelt werden, z. B. Blätter, Blüten, Früchte und Steine, und kann später in der Forscherstation noch genauer unter die Lupe genommen und bestaunt werden.

Zwischendurch erkundigen wir uns immer wieder mal, ob die Kinder schon Bambus entdeckt haben oder fragen sie, ob eine von uns gezeigte Pflanze Bambus ist. In ca. 90 \% der Fälle entdecken die Kinder dann schon von weitem den Bambuswald und sind oft ganz fasziniert,

Abb. 5 (oben): In der „Forscher-Station“.

Abb. 6 (Mitte): Der Riesen-Bambus (Dendrocalamus giganteus) im Tropicarium wird erkundet.

Abb. 7 (unten): Bestandteil jedes Programms ist das Eintopfen einer Pflanze in der Gärtnerei des Palmengartens. 
wie groß der Bambus ist und welche Farben die Halme haben können: grün, grün-gelb gestreift oder fast schwarz. Damit sie ihre Entdeckung festhalten können, bekommen sie die Möglichkeit, zu zweit auf einem kurzen Weg durch den Bambuswald ungestört den Bambus zu fotografieren, der ihnen am besten gefällt. Die Kinder gehen dabei durchgehend sehr vorsichtig mit den kleinen Digitalkameras um. Unsere erste Forscher-Reise endet am Spielplatz, wo die Kinder frei entscheiden, was und mit wem sie spielen.

Die „Kochkinder“, die sich morgens zum Kochen gemeldet haben, beenden ihren Aufenthalt auf dem Spielplatz etwas früher, um mit uns das Mittagessen für die Gruppe zuzubereiten. Dies ist eine weitere Besonderheit in unserem Projekt. Das Mittagessen ist in den Tagesablauf integriert, jeden Tag können bis zu fünf Kinder am Kochen teilnehmen, ein wichtiger und beliebter Aktionspunkt! Aus Zeitgründen können wir nur einfache Gerichte kochen, aber an deren Zubereitung werden die Kinder konsequent beteiligt.

Nach dem gemeinsamen Essen haben die Kinder die Möglichkeit, zwischen vielen Aktivitäten zu wählen. Sie können in der Bauecke bauen, die vorhandenen Spiele ausprobieren oder die Forscher-Station besuchen und sich dort unter Anleitung z. B. diejenigen Dinge genauer unter dem Binokular anschauen, die sie auf der Forscherreise gesammelt haben, oder die in der Sammlung vorhanden sind. Oft schließt sich noch ein kurzer Gang durch das Tropicarium an. Zum Abschluss des ersten Tages wird noch das „Märchen vom Bambusprinzen“ erzählt, der verzaubert in einem hohlen Bambushalm lebt, bis ihn ein Mädchen von seinem Zauber erlöst, als sie den Bambus schneiden will. Durch diese Geschichte, bei der wir auch einen RiesenbambusHalm aufklappen und dabei einen kleinen asiatischen Baby-Prinzen entdecken, wird den Kindern noch einmal deutlich, dass der Halm innen Hohlräume hat.

Abb. 8: Herstellung eines Rollbilds; oben sind als Umriss die chinesischen Zeichen für Bambus vorgegeben.

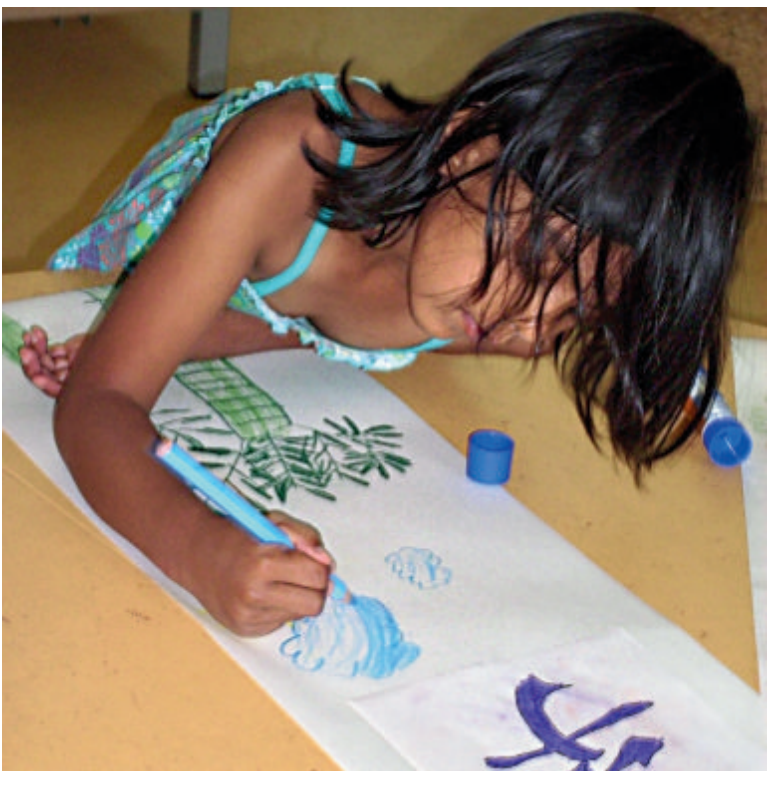

\subsection{Zweiter Tag}

Der zweite Tag beginnt wieder in der GesprächsEcke. Die Fotos, die die Kinder am ersten Tag gemacht haben, zeigen wir mit Hilfe eines Beamers groß an der Wand und wiederholen dabei die wesentlichen Kennzeichen des Bambus. Anhand einer Bilderserie zeigen wir den Kindern, welche Tiere von und im Bambus leben, selbstverständlich auch den Pandabären, der sich vorwiegend von Bambus ernährt und auf diesen angewiesen ist.

Die anschließende Forscher-Reise geht nun in das Tropicarium, diesmal mit dem Auftrag, den Riesen-Bambus (Dendrocalamus giganteus) zu finden. Auch hier entdecken die Kinder den Bambus sicher und können auch erklären, woran sie ihn erkannt haben. Wenn wir Glück haben, ist auch ein kleiner Bambus-Schössling zu erkennen. An einer Messlatte, die neben den Bambus-Spross gestellt wurde, können wir ablesen, wie schnell der Bambus in die Höhe schießt, nämlich an einem Tag bis zu $40 \mathrm{~cm}$. Wieder haben die Kinder die Gelegenheit, „ihren“ Bambus zu fotografieren. Eine mitgebrachte Packung mit Bambus-Sprossen wird betastet. Hierbei erkennen die Kinder, dass die jungen Sprosse noch weich sind und eine andere Farbe haben als die Halme und der ausgewachsene Spross. Ein WokGericht mit Bambus-Sprossen steht heute auf dem Speiseplan und ermöglicht es den Kindern, Bambus zu kosten. Wie jeden Tag endet die Forscherreise auf einem der Spielplätze oder bei Re- 


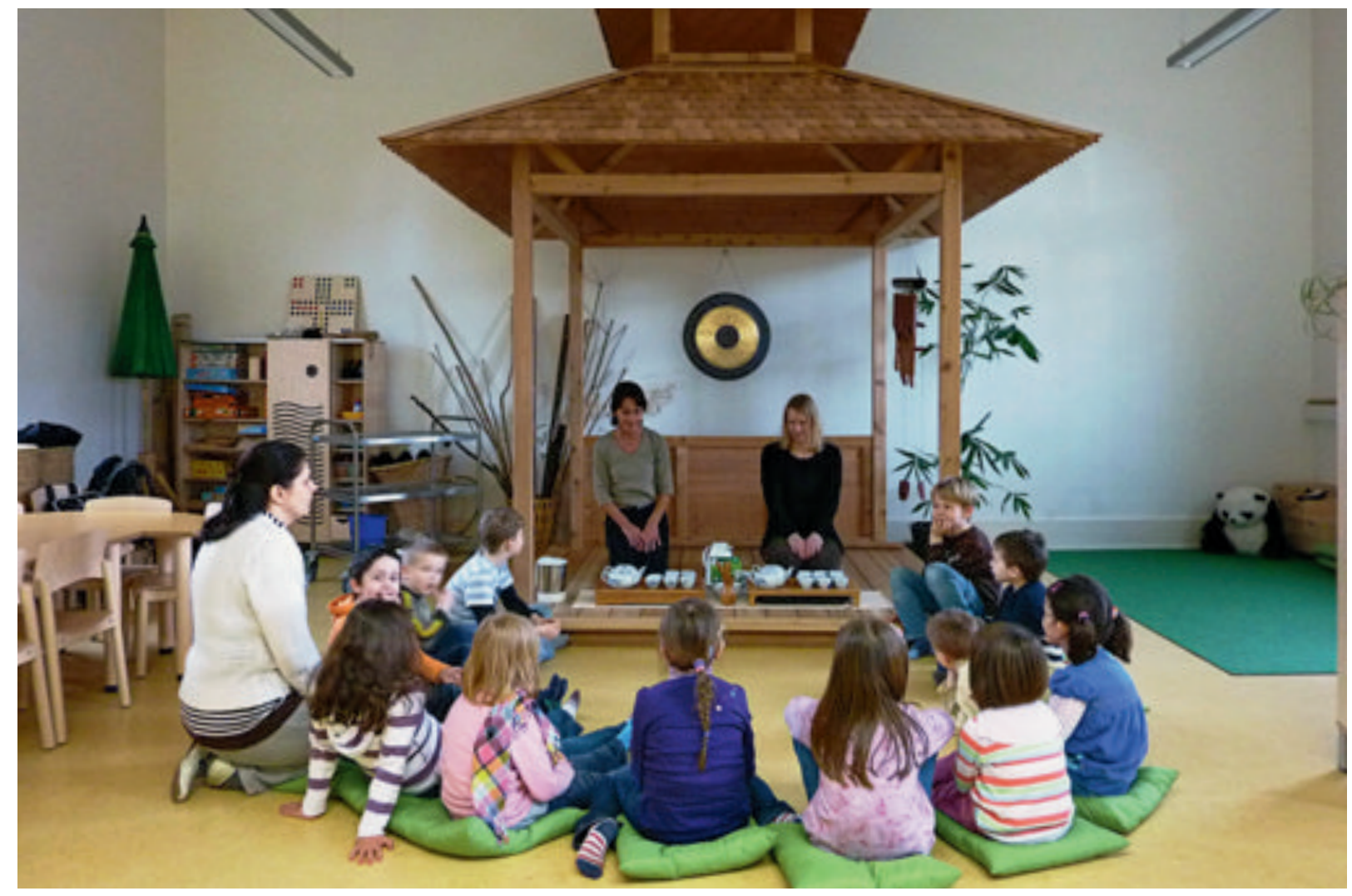

genwetter in der Einrichtung. Der Nachmittag ist von zwei Aktionen bestimmt. Wir teilen die Kinder in zwei Kleingruppen, wobei jede Gruppe an beiden Aktionen im Wechsel teilnimmt.

Aktion 1: Topfen einer kleinen Pflanze (bei passender Jahreszeit einen kleinen Bambus) in der Gärtnerei des Palmengartens.

Aktion 2: Herstellung eines asiatischen Rollbildes, das oben und unten durch Bambusstangen verstärkt wird.

Die Einteilung in Kleingruppen ist von Vorteil, da so intensiver auf die Bedürfnisse der einzelnen Kinder eingegangen werden kann. Zum Abschluss des zweiten Tages gibt es die "Geschichte vom Bambus, der zum Lebensretter wird“, weil man aus ihm Wasserrohre bauen kann, die ein ausgetrocknetes Reisfeld mit Wasser versorgen. Der Bambus stirbt dabei aber nicht, da aus seinem Wurzelstock immer wieder neue Halme nachwachsen können.

\subsection{Dritter Tag}

Auch am dritten Tag werden zuerst die Erlebnisse vom Vortag anhand der Fotos rekapituliert -
Wurzeln und Wachstum des Riesen-Bambus. Heute schließt sich dann eine Bilderserie an, in der verschiedenste Gegenstände gezeigt werden, die aus Bambus hergestellt werden: Häuser, Körbe, Pinsel, Baugerüste und sogar Fahrräder. Den Kindern wird dabei immer die Möglichkeit gegeben, zu raten, um was für einen Gegenstand es sich handeln könnte.

Das historische Palmenhaus ist Ziel der dritten Forscher-Reise. Auf dem Weg dorthin werden verschiedene Bereiche des Palmengartens bereist: Steingarten, Grotte, Wasserfall, Großer Weiher und Rosengarten - für die Kinder eine abwechslungsreiche und spannende Reise. Nachdem die Kinder die Höhe und Größe des Palmenhauses erfasst haben, bekommen sie einen Forscher-Auftrag, der gar nicht so einfach ist. Sie sollen im Palmenhaus den einzigen Standort finden, an dem Bambus wächst. In zwei Kleingruppen mit je einer Erzieherin geht es auf die Suche, ausgestattet mit Lageplan, Bambus-Halm und Digitalkamera. Da auch manche Palme oberflächlich

Abb. 9: Tee-Zeremonie mit Bambus-Tee zum Abschluss der drei Bambus-Tage. 
dem Bambus ähnelt, entwickeln sich oft kleine Diskussionen, aber meist wird der Bambus doch entdeckt.

Nach dem Mittagessen gibt es den letzten Auftrag: „Sucht Gegenstände in den Räumlichkeiten, die aus Bambus hergestellt sind!" Dabei werden von den Kindern die unterschiedlichsten Körbe, Kerzen, Schalen, Dekorationsartikel und Musikinstrumente zusammengetragen. Bei den Schalen stellt sich die Frage, wie aus einem zylindrischen harten Halm solche runden und ovalen Formen hergestellt werden. Die vielfältigen Ideen der Kinder werden gesammelt. Anhand einer Fotoserie und Zwischenprodukten erfahren die Kinder vom aufwendigen Herstellungsprozess. Gerade die Erzieherinnen und Erzieher sind oft erstaunt, wie wenig diese zeitaufwendig erarbeiteten Gegenstände bei uns kosten.

Zum Abschluss und Ausklang der drei Tage finden wir uns noch zu einer chinesischen TeeZeremonie zusammen, die wieder vieles mit Bambus zu tun hat und vor allem auch mit Bambus-Tee zubereitet wird. Die Kinder genießen den ruhigen und für sie sehr ungewöhnlichen Ablauf der Tee-Zeremonie.

\section{Resümee}

Die Erfahrungen, die wir seit einem Jahr gesammelt haben, zeigen, dass Kindergartenkinder immer wieder bereit sind, sich mit großem Interesse und hoher Motivation einem Thema zu widmen.

Der häufige Methodenwechsel, die Einbeziehung der Kinder als Akteure, die vielseitigen Wiederholungen, bei denen auch neue Begriffe erfahren werden, das spontane Eingehen auf kindliche Interessen sind nur einige Punkte für das Gelingen des Kinderangebotes. Dieses vielfältige Angebot ermöglicht, dass jedes Kind etwas findet, was es besonders interessiert. Grundvoraussetzung ist jedoch die Wertschätzung des Kindes. Sich wohlfühlen, zurechtfinden, wahrgenommen, ermuntert, bestätigt werden sind die grundlegende Basis auf dem Weg zum neugierigen Entdecken und Lernen.

Diese Programme mit den Kindern umfassen aber nicht unser gesamtes Angebot. Speziell für die begleitenden Erzieherinnen und Erzieher bie- ten wir Vor- und Nachbereitungsseminare an, in denen das Angebot und die angewandten $\mathrm{Me}-$ thoden vorgestellt, Fragen zum jeweiligen Pflanzen-Thema beantwortet sowie der Ablauf des Drei-Tages-Angebotes reflektiert werden. Wichtig ist uns außerdem die Einbeziehung der Eltern. Dazu laden wir mehrere Gruppen zusammen zu einem Aktionsnachmittag ein, an dem die Kinder ihre Eltern und Geschwister mitbringen können und ihnen z. B. bei einer Rallye durch den Palmengarten zeigen, was sie in den drei Tagen entdeckt, beobachtet, erlebt und gelernt haben.

Genauere Ergebnisse über die Nachhaltigkeit der Bildungsangebote werden im Rahmen einer begleitenden Evaluation durch die Universität Frankfurt erzielt werden. Hierzu finden Befragungen der beteiligten Kinder, Erzieherinnen und Erzieher und Eltern statt. Um das Erlernte noch weiter anzuwenden, haben wir mit dem Botanischen Garten und dem Zoo Frankfurt Kooperationen angebahnt, in deren Rahmen die Kinder bei einer Führung das jeweilige Thema noch um eine Facette erweitern können. So wird im botanischen Garten das Thema „asiatischer Bambus und einheimische Gräser" und im Zoo Frankfurt das Thema „Bambus als Lebensraum, Nahrung und Tarnung in der Tierwelt" angeboten.

\section{Literatur \\ STOlTEnBerG, U. 2008: Bildungspläne im Elementarbe- reich. Ein Beitrag zur Bildung für nachhaltige Entwick- lung? Eine Untersuchung im Rahmen der UN-Dekade „Bildung für nachhaltige Entwicklung“. \\ Deutsche UNESCO-Kommission. - Bonn.}

\section{Weiterführende Literaturempfehlungen}

LAEWEN, H.-J., LAEWEN, B. A. 2002: Bildung und Erziehung in der frühen Kindheit. - Weinheim, Basel, Berlin. LAeWen, H.-J., Andres, B. (Hrsg.) 2002: Forscher, Künstler, Konstrukteure. Werkstattbuch zum Bildungsauftrag von Kindertageseinrichtungen. - Neuwied, Kriftel, Berlin. SCHÄFER, G. E. (Hrsg.) 2004: Bildung beginnt mit der Geburt. - Weinheim, Berlin.

SCHÄFER, G. E. 1995: Bildungsprozesse im Kindesalter. Selbstbildung, Lernen und Erfahrung in der frühen Kindheit. - Weinheim. 